\title{
Modeling Peripersonal Action Space for Virtual Humans Using Touch and Proprioception
}

\author{
Nhung Nguyen, Ipke Wachsmuth \\ Faculty of Technology \\ Bielefeld University \\ 33594 Bielefeld, Germany \\ nnguyen, ipke@techfak.uni-bielefeld.de
}

\begin{abstract}
We propose a computational model for building a tactile body schema for a virtual human. The learned body structure of the agent can enable it to acquire a perception of the space surrounding its body, namely its peripersonal space. The model uses tactile and proprioceptive informations and relies on an algorithm which was originally applied with visual and proprioceptive sensor data. In order to feed the model, we present work on obtaining the nessessary sensory data only from touch sensors and the motor system. Based on this, we explain the learning process for a tactile body schema. As there is not only a technical motivation for devising such a model but also an application of peripersonal action space, an interaction example with a conversational agent is described.
\end{abstract}

\section{Introduction and Related Work}

In order to carry out sophisticated and challenging interaction tasks in a spatial environment like a virtual world, one requisite is to perceive how far away objects in the peripersonal space are in relation to the protagonist's own body. The peripersonal action space is the space which immediately surrounds our body, in which we can reach, grasp and manipulate objects with our limbs without leaning forward. The ability of virtual humans to perceive and adapt to their peripersonal space enables them to manipulate and also to avoid objects while moving their limbs through this space. Additionally, it raises more interpersonal interaction possibilities with other agents and also with human partners.

Since virtual worlds are fast-changing and becoming more demanding, we go along with Magnenat-Thalmann and Thalmann who stated that it is important to enable virtual humans to have a realistic perception of the environment surrounding them, and to make them aware of it by building touch, vision and proprioception modeled on humans' perception [9]. Conde and Thalman [1] presented a model which emphasizes the role of a unified agent perception to establish a cognitive map of the Virtual Environment. This perception model integrates multiple virtual sensors and enables an autonomous virtual agent to predict object locations in an agent-centered vision space. In our definition we also consider the agent's peripersonal space as being centered on the agent, but spanned by its body. It enables the agent to predict object locations in reaching space. 
In humans the representation of peripersonal space is intimately connected to the representation of the body structure, namely the body schema [6]. The most comprehensive definition of the body schema, as a neural representation, which integrates sensor modalities, such as touch, vision and proprioception, was provided by Gallagher [3]. This integration or mapping across the different modalities is adaptive and explains phenomena like tool use as an integration of tools into the body schema [10]. Learning of body schema is very versatile. We can not only learn configurations of a body structure, but according to Holmes and Spence [6] it also supports learning of the space surrounding the body.

Learning a body schema can also be of great interest for developing advanced virtual characters in computer games. Especially games, which integrate user generated game content become more and more popular since they offer more diverse game courses. One example is the game Spore 1 where players are allowed to create characters and creatures according to their own imagination. The player can, for instance, add several legs, arms, wings and other body parts to the creature. Its locomotion changes with the added parts, but is predefined in the game. For these new kinds of games, where the body structure of the characters are built by the player, learning the kinematic functions of body structures could, in the future, lead to smoother and more lifelike movements and behaviour.

To our knowledge, work on learning reaching space for embodied agents has yet been done isolated from body schema acqusition ([7], [4]). So far, this topic has been dominated by robotics researchers in order to build adaptive body schemas. For example, Yoshikawa et al. [12] presented work on how a robot learns a body schema by mapping visual, proprioceptive and tactile sensor data using a cross-modal map. Fuke et al. [2] used the same modalities for learning a representation of a simulated face, using a self-organizing map. One crucial argument for an adaptive representation of the body structure are possible changes in the body configuration of humanoid robots. This method can replace laborious, manual adjustments. Although the topic is mainly treated by roboticists and has yet not been applied to virtual agents, we want to point out how learning a body schema can also further the design of virtual humans and characters.

In this paper we will show how to model a tactile body schema for a virtual agent and how this can be used to build a representation of its peripersonal action space. Both approaches, as far as we can see, have not been presented in previous works. Preconditions for the tactile body schema are our work on building touch sensors and motor abilities for a virtual agent. For learning a body schema, we base our computational model on the algorithm proposed by [5]. Unlike their approach, we will not use vision but will feed touch and joint information into the algorithm, in order to learn a tactile body schema, which therefore gets along without any visual information. Combining it with motor abilities, the virtual human is able to perceive its peripersonal space. This can also be regarded as a proof of concept which shows that the spatial representation of the body and peripersonal space, respectively, are not bound to visual information, since congenitally blind people are also able to perceive their peripersonal space. Therefore, everytime the agent perceives tactile stimuli on a certain body part (e.g. the left upper arm), coming from objects within his reaching space, the learned body represen-

\footnotetext{
${ }^{1} \mathrm{http}: / / \mathrm{www}$. spore.com
} 
tation provides the spatial relation between the object and any other body part (e.g. the right hand). This enables the agent to carry out adequate movements, like avoiding or reaching an object, by taking the objects' spatial location into account.

The remainder of this paper is organized as follows. In the next section, we describe how virtual sensors were realized and prepared in order to feed our model of tactile body schema, described in Section 3 In Section 4 we present a demonstration scenario in which the tactile body schema can make an impact on peripersonal space. In Section 5 we briefly discuss how the properties of the model account for designing intelligent virtual humans on the one hand and virtual characters and creatures in computer games on the other hand. Finally, in Section 6 we give a brief conclusion and an outlook on future work concerning the interaction abilities of our virtual human Max.

\section{Touch Perception and Proprioception for a Virtual Human}

In this section we will first describe in general how a virtual sense of touch was realized for the virtual human Max [11]. In order to feed our computational model which we present in Section 3, we had to prepare the sensory data from the touch modality and complement it with sensory data from the motor modality. Therefore, in this section we specify which informations are extracted from the touch sensors and from the motor system to feed the model.

The touch receptors were developed and technically realized for Max's whole virtual body. These receptors allow for differentiating between different qualities of tactile stimulation. Findings from studies on the human tactile systems were incorporated to build an artificial sense of touch for Max, which is conceived not only for virtual but for artificial agents in general. In our work on modeling and realizing passive touch for Max's whole body, each tactile stimulation is associated with characteristics, namely, where on Max's body it was applied and what kind of tactile stimulation it was, e.g. stroking or tapping.

Max has a segmented body, i.e. his virtual graphical embodiment consists of several geometry parts. Around every geometry representing a limb of Max's body, 17 proximity geometries were added forming a "proximity aura" (see Figure 1, middle). This allows for predicting when an object in the VR environment is approaching Max's body. In humans, the somatosensory modality is represented in body-part-centered reference frames [6]. This aspect is also modeled by the virtual proximity auras and therefore they enable Max to identify the body part an object may be going to touch. Below the proximity aura, the surface of Max's body is covered with a virtual "skin". The virtual skin consists of flat quadrangle geometries varying in size, each representing a single skin receptor (see Figure 1, right). Altogether the virtual skin consists of more than 200 virtual skin receptors. The receptors are located on the body in neighborhoods, which are represented in a somatotopic map (similar to the map in the human brain).

This representation encodes the information which body limb a virtual skin receptor is attached to, and it allows to determine, in a fine-grained way, where Max is being touched. Depending on the location on the body, a tactile stimulation can thus be interpreted differently. Instead of different kinds of skin receptors as in the human skin, only one kind of virtual skin receptor is utilized for Max, for it is sufficient to discriminate 


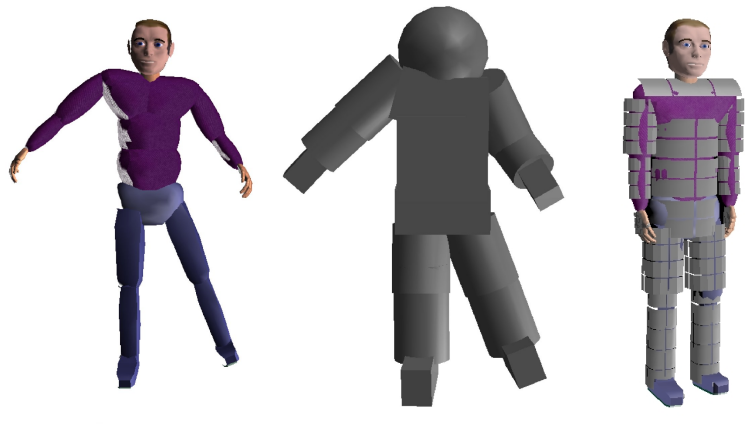

Fig. 1. Max's segmented body (left) with proximity geometries allowing for predicting touching of objects and identifying the touch receptor's corresponding limb (middle). Max's virtual body covered with over 200 virtual skin receptors (right).

between different tactile stimulations. Every object that is graphically represented in our VR environment can cause tactile stimuli on Max's virtual skin.

Any geometry's collision with a skin receptor is regarded as tactile stimulus. This also includes skin receptors colliding with each other which is crucial for identifying self-touch. Specific stimulation patterns arise from the temporal and local spatial changes connected to the stimulation. When a stimulus is, e.g., moving continuously over the skin, neighboring receptors are responding successively over time. This temporal information along with the spatial information about each triggering receptor, extracted from the somatotopic map, allows for classifying the stimulation as a continuous touch movement about the respective body parts. A central component, that fuses these stimulations of the receptors into a coherent touch sensation, forms our touch sensor.

So far, the classification of the different tactile stimulations depend on a somatotopic map, which was constructed manually. The skin receptor geometries are each assigned to a unique ID and are organized in 8-neighborhoods. That is, for each skin receptor ID, there exists an entry in the map, which contains the skin receptor IDs of the eight neighbors. Additionally, each skin receptor is assigned to a unique body limb, therefore the receptors' locations and distances are not centrally encoded, which reflects the already mentioned body-part-centered representation of the human touch modality. In the computational model described in Section 3 , for each skin receptor, the touch sensor provides the assignment to the unique body limb and its position in the frame of reference (FOR) of that corresponding limb.

In addition to the artificial sense of touch, we need proprioceptive information about Max's body. In humans, proprioception is the sense of the orientations and positions of the limbs in space. It is important for perceiving motor control and body posture. We will refer to it, as commonly used in embodied agents, as the angle configuration of the joints in Max's body skeleton. The virtual agent's body has an underlying anthropomorphic kinematic skeleton which consists of 57 joints with 103 Degrees of Freedom (DOF) altogether [8]. Everytime Max is executing a movement, the joint angle infor- 


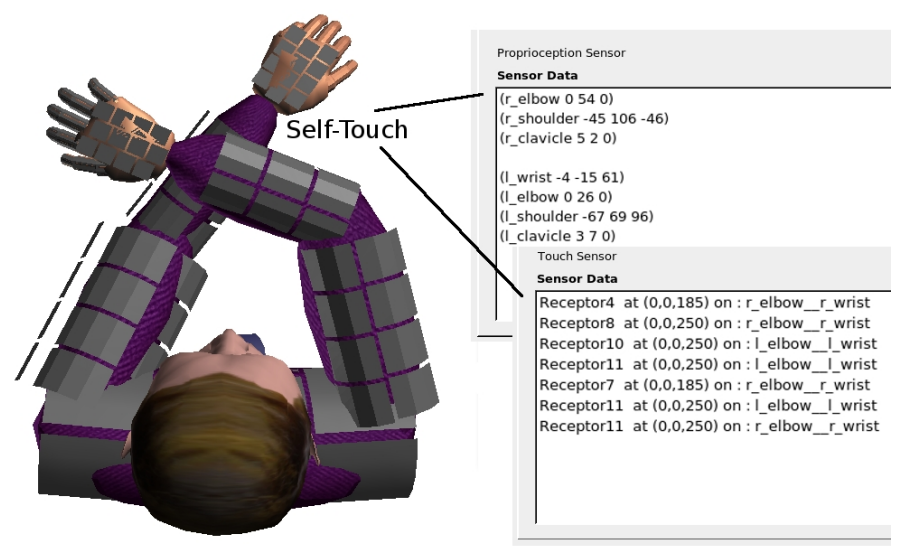

Fig. 2. Tactile body schema learning: For each random posture, sensory consequences are output by the sensory systems. The touch sensor provides an ID of the receptor, the limb it is attached to, and the position in the frame of reference (FOR) of the corresponding limb. Angle data for the involved joints are output by the motor system, representing the proprioceptive information.

mations of the involved joints are output. Synchronously with the tactile informations, the proprioceptive informations can be observed. In Figure 2 we can see the data for a sample posture, where Max is touching his own arm. In the next section we will explain how these input data can be integrated to form a body schema.

\section{A Computational Model of Peripersonal Space Based on a Body Schema}

In this section we present our model on how to learn a tactile body schema for our virtual human Max. The idea is to integrate tactile and proprioceptive information from his virtual body. In a first step, Max executes random motor actions resulting in random body postures. For each posture he perceives proprioceptive data from his joints and tactile stimuli when touching himself (see Fig. 2). The model integrates input data given by the touch sensors and joint angle data given by the proprioception sensors described in Section 2,

\subsection{Tactile Body Schema for Peripersonal Space}

The tactile body schema, learned by the virtual human, in our model depends on its sensory system and FOR transformations associated with the sensory input coming from the touch and proprioception sensors. For our purposes, that is, perceiving and acting in peripersonal space, a tactile body schema is sufficient. We do not need a precise representation of the physical properties of the body, rather we need the kinematic structure 


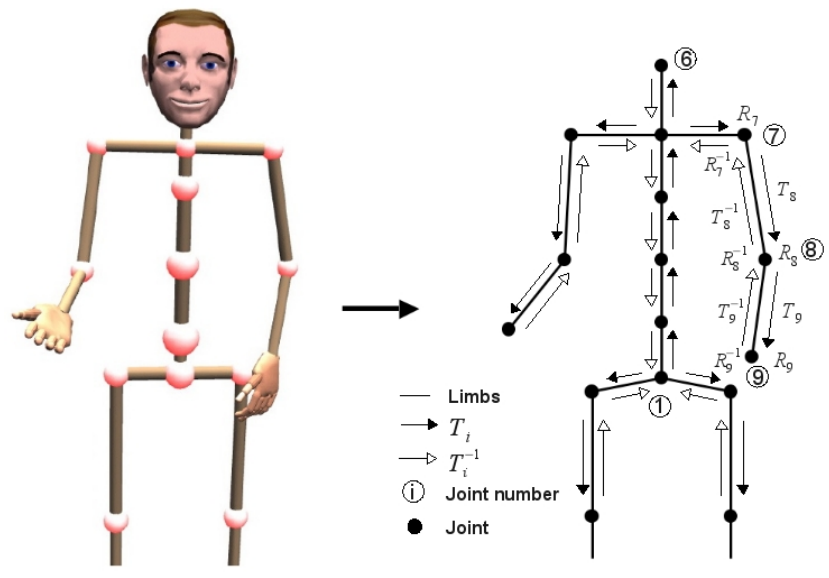

Fig. 3. Kinematic tree (right) representing Max's body skeleton (left). Following an edge in direction to the root node representing the hip joint (joint 1), a FOR transformation $\mathbf{T}_{i}$ and a rotation $\mathbf{R}_{i}$ associated to the respective joint $\mathrm{i}$ (numbers are free chosen) have to be carried out, in the other direction we use the inverse FOR transformation $\mathbf{T}_{i}^{-1}$ and rotation $\mathbf{R}_{i}^{-1}$. Example: The following composition transforms a FOR centered on joint 7 to a FOR centered on joint 6 (joint 5 is located between them): $\mathbf{R}_{6}^{-1} \circ \mathbf{T}_{6}^{-1} \circ \mathbf{T}_{7}$ $\circ \mathbf{R}_{7}$.

and functions of the body for controlling and predicting the sensory consequences and movements with regard to tactile stimulations coming from objects located within the reaching space.

Given a proprioceptive input, together with input from a certain touch receptor to a corresponding particular posture, the body schema can predict proprioceptive sensor consequences for other touch receptors. This can be used to generate a movement, corresponding to the proprioceptive data. The following example makes its utilization more clear. Let us assume a virtual human accidentally touches an object located in its peripersonal space with its right upper arm. Note that there is no visual information. In order to "touch" the same object with the left hand, the agent needs to know how to move the left arm (see Section 4).

\subsection{Learning a Tactile Body Schema}

We follow Hersch et al. [5] by considering the body schema as a tree of rigid transformations. In our case this kinematic tree is prescribed by the skeleton of the virtual human Max. In this tree each node corresponds to a joint in Max's skeleton and each edge corresponds to a limb between two joints (see Figure 3 ). That means, the number of joints linked in their respective order with the number of limbs are represented in the kinematic tree, but not the joint orientation and position. In our model the touch receptors are attached to the limbs (see Section 2) and their position is represented in 


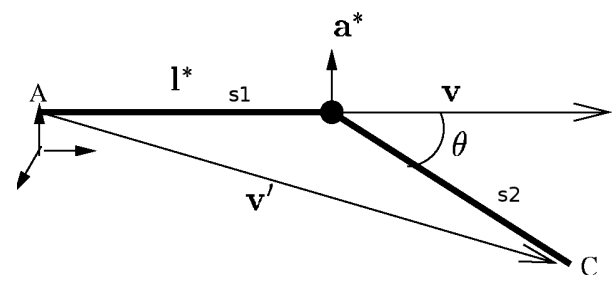

Fig. 4. Example of a single segment manipulator with $\mathrm{A}$ as the origin, $\mathrm{C}$ as the end effector, s1 as the proximal, and s2 the distal segment. A rigid transformation is here parameterized by vectors $\mathbf{l}(*$ joint position) and $\mathbf{a}$ (*rotation axis). Along with the known angle $\theta$ and given a vector $\mathbf{v}$ in the FOR of s2, and its transform $\mathbf{v}$ ' in the FOR of $\mathrm{s} 1, \mathbf{l}$ and $\mathbf{a}$ can be adapted online (after [5], Fig.2).

the limb's FOR. In the kinematic tree representation, the touch receptors can therefore be represented as located along the edges.

Following an edge from one joint to another is associated with a FOR transformation which transforms the FOR centered on one joint to the FOR centered on the other joint. Therefore, following any path linking one joint to another represents a kinematic chain. Max's skeleton prescribes the hierarchy of the FOR transformations. This determines whether a normal or inverse FOR transformation has to be carried out along a kinematic chain. Figure 3 shows the transformation hierarchy with the hip joint as root node in the kinematic tree representing Max's skeleton. The kinematic chains transform positions and orientations from the FOR centered on the different joints. Since the touch receptors are attached to the limbs, we can transform the position for one touch receptor, given in the FOR of the corresponding limb, into any other touch receptor position also given in the FOR of its corresponding limb.

So far, we use the number of joints and the hierarchy of Max's skeleton as prior knowledge about his body structure. However, what is not yet known is the position and orientation of these joints which also determine the limb lenghts. This is where the algorithm proposed by Hersch et al. [5] comes in. We can use the algorithm straightforward, since it provides a new and general approach in online adapting joint orientations and positions in joint manipulator transformations. Our challenge in using this algorithm is the adaptation to a case different from the one it was originally applied to. In our case we do not use visual and joint angle data but instead, replace all visual by tactile information in order to update all the rigid transformations along the generated kinematic chains. As far as we know, this case has not been presented before.

Here we will only sketch the key ideas of the algorithm and then describe how they can be adapted for our purposes. The algorithm deals with the problem of having a single segment manipulator as shown in Figure 4 A rigid transformation carried out by the manipulator is parameterized by unknown vectors $\mathbf{I}$ (the joint position) and $\mathbf{a}$ (the unit rotation axis) and a known rotation angle $\theta$. The vectors $\mathbf{a}$ and $\mathbf{l}$ can be adapted, so that they match the rigid transformation. This is done by means of a given vector $\mathbf{v}$ in a FOR attached to the distal segment s2, and its given transform $\mathbf{v}$ ' in a FOR attached to 
segment $\mathrm{s} 1$ and the rotation angle $\theta$. A gradient descent on the squared distance between $\mathbf{v}^{\prime}$ and its guessed transform vector $\mathbf{l}+\mathbf{R}(\mathbf{v})$ is used in order to update the guesses of the vectors $\mathbf{a}$ and $\mathbf{l}$.

Having sufficient examples of positions (values for $\mathbf{v}$ ) given in the FOR of segment s2 and the corresponding positions (values for $\mathbf{v}^{\prime}$ ) given in the FOR of segment s1, it is possible to adapt the joint positions and orientations. For an adaptation of multisegmented manipulators the simulated transform vector $\mathbf{l}+\mathbf{R}(\mathbf{v})$ was replaced by a transformation $\mathscr{T}(\mathbf{v})$, which contains the transformations along the kinematic chain of the multisegment manipulator. In our case the kinematic chains can be generated using the kinematic tree representing Max's body skeleton (see Figure 3). The rotation axes and translation vectors of joint $i$ can then be updated by using the equations (1) and (2) (taken from [5]) with a small positive scalar $\varepsilon$, and rotation matrix $\mathbf{R}_{i}$ of axis $\mathbf{a}_{i}$ and angle $\theta_{i}$ for joint $i$.

$$
\begin{gathered}
\Delta \mathbf{l}_{i}=\varepsilon\left(\mathbf{v}_{n}^{\prime}-\mathscr{T}\left(\mathbf{v}_{n}\right)\right)^{T} \prod_{j=1}^{i-1} \mathbf{R}_{j} \\
\Delta \mathbf{a}_{i}=\varepsilon\left(\mathbf{v}_{n}^{\prime}-\mathscr{T}\left(\mathbf{v}_{n}\right)\right)^{T}\left(\left(\prod_{j=1}^{i-1} \mathbf{R}_{j}\right) \frac{\partial}{\partial \mathbf{a}_{i}}\left(\mathbf{R}_{i}\left(\mathbf{T}_{i+1} \circ \mathbf{R}_{i+1} \ldots \circ \mathbf{T}_{n} \circ \mathbf{R}_{n}\left(\mathbf{v}_{\mathbf{n}}\right)\right)\right)\right)
\end{gathered}
$$

In order to use the algorithm, we have to start with an onset body schema which is an initial guess of Max's target body schema. It is described on the one hand by known parameters and on the other hand by initially guessed parameters. The number of joints and their hierarchical order are determined by the kinematic tree of Max's body skeleton, described above. The parameters which are not known yet are the joint orientations and their positions, determining the body segment lenghts. Thus we choose the orientations randomly and assign the segment lengths with small values.

For modeling peripersonal space we start with learning the schema for Max's torso, which includes all nodes above the hip joint to the wrist joints. For a first approach we do not use the joints in the hands, since a sophisticated touch sensation for the hands and fingers (with over 30 receptors per hand) may not be nessessary for reaching space. We then have to choose random joint angle configurations for the torso. For each randomly chosen posture, the agent will carry out a motion which leads to the joint angle configuration and then stop. If skin receptors are touching each other during the motion, Max will immediately stop moving in order to avoid the case of passing through the graphical limbs. The originally assumed joint angle configuration will then be discarded, and instead, the current joint angle data is taken and the resulting sensor data is processed. The input for the algorithm are the positions of two touch receptors touching each other in the FOR of their corresponding limbs, both provided by the touch sensor (see Figure 5). Interestingly, both positions can take over the role of the input vectors $\mathbf{v}$ and $\mathbf{v}$ ' for the Equations 1 and 2 This is also illustrated in the pseudo code for the tactile learning process in Algorithm 1. Additionally, the angle values of the joints involved in the current posture are input to the algorithm. It then takes the sensor data for updating its guesses of the joint orientations and positions of the involved kinematic chain. 


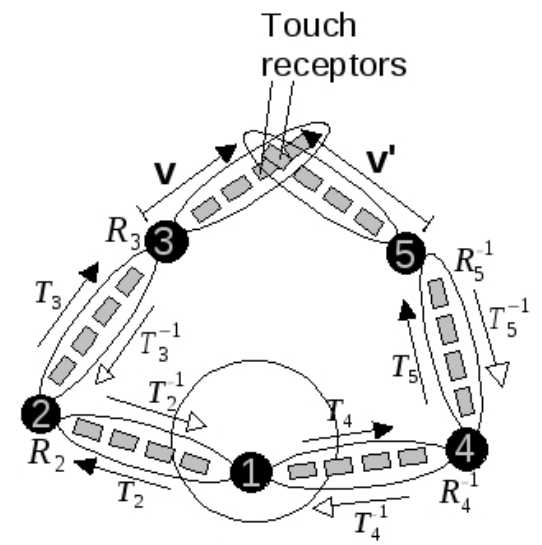

Head

Fig. 5. Schema of Max touching himself (notation following Figure 3). The following composition transforms the position $\mathbf{v}$ (given in the FOR centered on joint 3 ) of a touch receptor into the FOR centered on joint 5: $\mathbf{R}_{5}^{-1} \circ \mathbf{T}_{5}^{-1} \circ \mathbf{R}_{4}^{-1} \circ \mathbf{T}_{4}^{-1} \circ \mathbf{T}_{2} \circ \mathbf{R}_{2} \circ \mathbf{T}_{3} \circ \mathbf{R}_{3}$. Note that retracing the same chain in the opposite direction transforms the position of the other touch receptor $\mathbf{v}$ ' (given in the FOR centered on joint 5) into the FOR centered on joint 3 .

In the adaptation process the idea is to use the algorithm two times for each posture (see Algorithm 1, Line 18-22). In a first process the transformation of the position $\mathbf{v}$ of one touch receptor is transformed into the FOR of the other touch receptor (Line 13). This is used to update the current body schema (Line 14-16), in a second pass the angles of the postures stay the same, but the kinematic chain linking the two touch receptors is retraced to transform the position $\mathbf{v}$ ' of the other touch receptor. Note that this "double-use" is only possible in the case of learning a tactile body schema.

After completion the learned body schema expectedly contains the kinematic functions derived from the sensory input. This can be used to control Max's movements with regard to tactile stimuli.

\section{Peripersonal Space in Interaction}

Based on the work presented in Section 2, we devised the computational model in Section 3 for building a body-representation for the virtual humanoid Max. This model can enable him to acquire a perception of his peripersonal space, i.e. the space immediately surrounding his body and which he can reach with his limbs. In this section we outline an interaction example in which peripersonal space can be utilized by the virtual human Max. Previous works on peripersonal space and body schema acquisition may lack the application and interaction possibilities, since the agents are often regarded as technical platforms. However Max is an embodied conversational agent and is primarily intended 


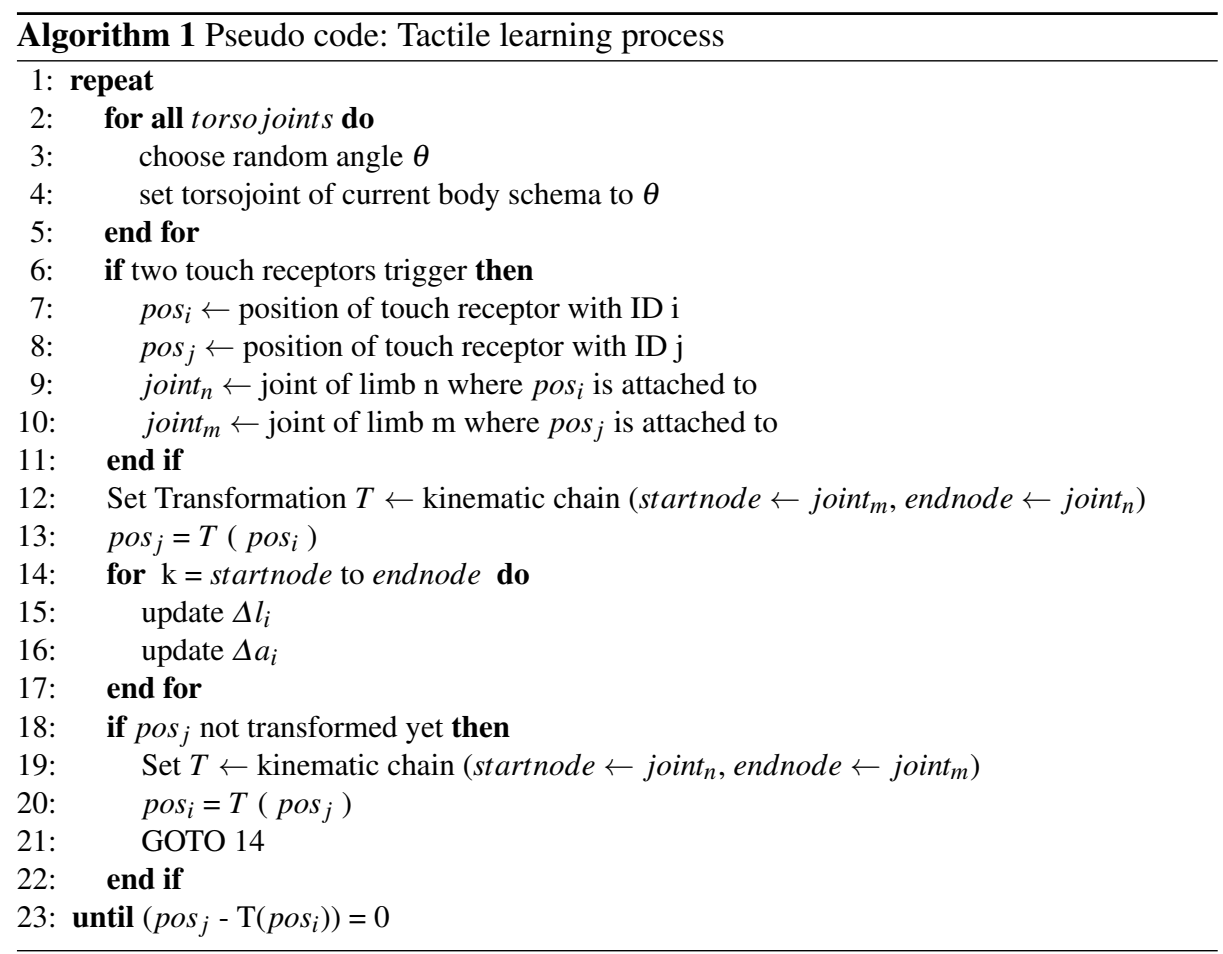

for interaction with human or other virtual partners, hence the application of peripersonal space can be shown in an example. In this example Max is standing at a table. In an interaction scenario Max could interact in a CAVE-like environment with a human partner as shown in Figure 6 .

In our test scenario, several objects are located on the table. Let's assume Max is (technically) "blindfolded". The interaction partner, aware of Max's inability to see, asks him to reach for an object near to his body. He then explores his peripersonal space with one hand. Depending on the object's location he might touch it with his hand but also with any other part of his arm, since it also has skin receptors. As soon as he touches it, the partner could ask him to carry out tasks, such as touching the object with the other hand or putting it as far from him as possible. The first task is supported by the tactile body schema which contains the kinematic transformations relating two touch receptors. This can be used to compute a movement to the respective position. The task of putting the object as far away as possible is an interesting aspect relating to peripersonal reaching space. It is conceivable that Max could also learn the kinematic function of leaning forward in order to extend his peripersonal space.

\section{Discussion}

Our model aims at learning a tactile body schema using touch and proprioception instead of vision and proprioception. Knowledge about the joint number and hierarchy 


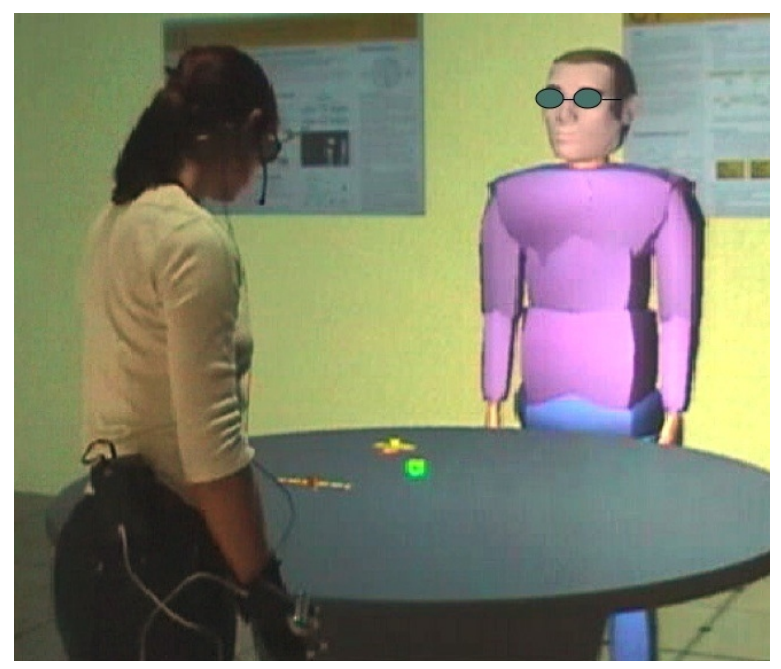

Fig. 6. Virtual agent Max with a human interaction partner standing around a table in a CAVE-like Virtual Reality environment. By means of his peripersonal space Max may perceive objects located on the table in front of him as near or far away from his body.

of Max's body skeleton is given in advance. This approach is important to produce the effectiveness of the used algorithm. Hersch et al. [5] for example argue that the kinematic structure in humanoids usually does not evolve over lifetime, limbs grow, but new joints do not appear. We agree with this opinion, but do not regard it as a contribution to ongoing neuroscience research of body schema acquisition. In fact we consider it as a contribution to learning kinematic structures for the special requirements of intelligent virtual agents like Max. In robots it is not easy to realize a sophisticated skin and it takes much longer to learn a body schema, since random explorations cannot be executed very fast. Due to these circumstances, a "virtual" simulation model of the robot is usually employed to learn the schema and afterwards is transferred to the physical robot. We want to point out that methods from other research fields which already incorporate "virtual bodies" are of special interest for the design of virtual humans. In Section 1 we mentioned the applications for virtual creatures in computer games. Body structure learning would enable the players to design creatures even with more unusual kinematic structures, not comparable to humanoid ones. In these cases, the skeleton is also predefined by the designer, therefore methods which take this pre-knowledge for learning lend themselves for an immediate use in character animation in computer games.

\section{Conclusion and Future Work}

In this paper, we proposed a computational model for building a tactile body schema for the virtual humanoid Max, which can enable him to acquire a perception of his 
peripersonal space. The proposed computational model uses tactile and proprioceptive informations and relies on an algorithm, which was originally applied with visual and proprioceptive sensor data. In order to feed the model, we presented work on obtaining the nessessary sensory data from touch sensors and the motor system. Based on this, we described the learning process for a tactile body schema. The next step in our work will be to test the proposed model for its online learning features.

As Max is a conversational agent, a possible example for using peripersonal space in interaction was shown. Subsequently the described interaction scenario could be used to study further research questions. In future work we will investigate how spatial perspective models of two agents can be aligned. In a cooperative interaction task, two artificial agents, or an agent and a human partner, jointly have to solve a problem by moving or manipulating (virtual) physical objects. In the shared space between the partners, one agent can use his peripersonal space representation (the space immediately surrounding the body) and map it onto the interaction partner. This representation could then be augmented with visual information. Based on the interlocutor's position and orientation, the agent can now infer the spatial perspective of the partner, by aligning the mapped peripersonal space representation of his own with position and orientation parameters of the other. This perspective model can help the agent anticipate actions performed, or hindrances encountered, by his partner. For example, when the agent sees an object which he infers to be hidden from the perspective of the other, the agent can move the object so the partner can see it. In a further step, the agent can develop a representation of interpersonal action space, i.e. the space between the two partners where their individual peripersonal space representations meet or overlap. A challenge is to develop an analogical spatial representation suited for peripersonal and interpersonal action space and, further, to devise methods for a dynamical alignment of interpersonal space representation when one or both interlocutors change their positions or body orientations.

\section{Acknowledgments}

This research is carried out in the context of the Collaborative Research Center 673 Alignment in Communication granted by the Deutsche Forschungsgemeinschaft. This paper is a preprint version of an article published by Springer-Verlag. The original publication is available at http://link. springer . com/chapter/10.1007/978-3-642-04380-2_ 11

\section{References}

1. T. Conde and D. Thalmann. An integrated perception for autonomous virtual agents: active and predictive perception. Journal of Visualization and Computer Animation, 17(3-4):457468, 2006.

2. S. Fuke, M. Ogion, and M. Asada. Body image constructed from motor and tactile image constructed from motor and tactile images with visual information. International Journal of Humanoid Robotics (IJHR), 4(2):347-364, 2007.

3. S. Gallagher. How the body shapes the mind. Clarendon Press, Oxford, 2005. 
4. C. Goerick, H. Wersing, I. Mikhailova, and M. Dunn. Peripersonal space and object recognition for humanoids. In Proceedings of the IEEE/RSJ International Conference on Humanoid Robots (Humanoids 2005), Tsukuba, Japan, pages 387-392. IEEE Press, 2005.

5. M. Hersch, E. Sauser, and A. Billard. Online learning of the body schema. International Journal of Humanoid Robotics, 5(2):161-181, 2008.

6. N. Holmes and C. Spence. The body schema and multisensory representation(s) of peripersonal space. Cognitive Processing, 5(2):94-105, 2004.

7. Z. Huang, A. Eliëns, and C. T. Visser. Is it within my reach? - an agents perspective. In Proceedings of Intelligent Virtual Agents, IVA 2003, pages 150-158, 2003.

8. S. Kopp and I. Wachsmuth. Synthesizing multimodal utterances for conversational agents. Comput. Animat. Virtual Worlds, 15(1):39-52, 2004.

9. N. Magnenat-Thalmann and D. Thalmann. Virtual humans: thirty years of research, what next? The Visual Computer, 21(12), 2005.

10. A. Maravita and A. Iriki. Tools for the body (schema). Trends in Cognitive Sciences, 8(2):7986, 2004.

11. N. Nguyen, I. Wachsmuth, and S. Kopp. Touch perception and emotional appraisal for a virtual agent. In Proceedings Workshop Emotion and Computing - Current Research and Future Impact, KI-2007, Osnabrueck, 2007.

12. Y. Yoshikawa, M. Yoshimura, K. Hosoda, and M. Asada. Visio-tactile binding through double-touching by a robot with an anthropomorphic tactile sensor. International Conference on Development and Learning, page 126, 2005. 